\title{
Effects of Process-Based Cognitive Training on Memory in the Healthy Elderly and Patients with Mild Cognitive Impairment: A Randomized Controlled Trial
}

\author{
Minyoung Shin ${ }^{1,2 *}$, Ahee Lee ${ }^{1,3 *}$, A Young Cho', Minam Son', and Yun-Hee Kim ${ }^{1,3,4 凶}$ \\ ${ }^{1}$ Department of Physical and Rehabilitation Medicine, Center for Prevention and Rehabilitation, Heart Vascular and Stroke Institute, \\ Samsung Medical Center, Sungkyunkwan University School of Medicine, Seoul, Republic of Korea \\ 2Department of Counseling Psychology, Yongmoon Graduate School of Counseling Psychology, Seoul, Republic of Korea \\ ${ }^{3}$ Department of Health Sciences and Technology, Sungkyunkwan University, Seoul, Republic of Korea \\ ${ }^{4}$ Departments of Medical Device Management and Research, Digital Healthcare, SAIHST, Sungkyunkwan University, Seoul, Republic of Korea
}

Objective This study investigated the effects of process-based cognitive training that targets working memory and cognitive control on memory improvement in healthy elderly individuals and patients with mild cognitive impairment (MCI).

Methods Forty healthy subjects and 40 patients with MCI were randomly assigned to either the intervention or control group. The intervention group received 12 sessions of designated cognitive training. The control group did not receive cognitive training. A memory test was administered pre-intervention, post-intervention, and 4 weeks after the intervention. Additional comprehensive neuropsychological tests were also administered including a depression scale questionnaire.

Results Performance in attention and working memory, which are directly related to the training domains, and global cognitive function were improved in the intervention group after training. In memory tests, interference by irrelevant stimuli was reduced and recognition memory was improved after the intervention. Furthermore, cognitive training ameliorated depressive symptoms. These training effects were not dependent on MCI status.

Conclusion Process-based cognitive training that targets working memory and cognitive control effectively improves memory processes including retrograde interference and recognition, as well as depressive symptoms associated with aging in healthy elderly individuals and patients with MCI.

Psychiatry Investig 2020;17(8):751-761

Key Words Aging, Mild cognitive impairment, Process-based cognitive training, Working memory, Cognitive control.

\section{INTRODUCTION}

Memory impairment is a main health concern associated with aging, and memory problems are common in healthy aging and in pathological aging such as mild cognitive impairment (MCI) or dementia. ${ }^{1,2}$ Cognitive decline naturally oc-

Received: October 2, 2019 Revised: February 7, 2020

Accepted: March 18, 2020

$\square$ Correspondence: Yun-Hee Kim, MD, PhD

Department of Physical and Rehabilitation Medicine, Center for Prevention and Rehabilitation, Heart Vascular Stroke Institute, Samsung Medical Center, Sungkyunkwan University School of Medicine, Departments of Medical Device Management and Research, Digital Healthcare, SAIHST, Sungkyunkwan University, 81 Irwon-ro, Gangnam-gu, Seoul 06351, Republic of Korea

Tel: +82-2-3410-2824, Fax: +82-2-3410-0388

E-mail: yun1225.kim@samsung.com

*These authors contributed equally to this work.

(a) This is an Open Access article distributed under the terms of the Creative Commons Attribution Non-Commercial License (https://creativecommons.org/licenses/bync/4.0) which permits unrestricted non-commercial use, distribution, and reproduction in any medium, provided the original work is properly cited. curs with aging in widespread cognitive domains including those associated with attention, memory, visuospatial, language, and executive function. ${ }^{3}$ Because memory problems are easily recognizable and an overt outcome of cognitive processes, any cognitive dysfunction is often considered a memory problem regardless of other dysfunctions in attention or executive function that can underlie inefficient memory functioning.

Memory training programs that teach mnemonic strategies have recently emerged to improve memory, and previously published studies indicate that such programs are effective in enhancing memory performance among older adults. ${ }^{4-6}$ However, some researchers have expressed concern that this strategy-based intervention that utilizes training with specific mnemonics has limited potential for generalization to other cognitive domains or to everyday functions. ${ }^{7}$ Moreover, such strategybased training was less effective for older adults compared to young adults; ${ }^{6,8}$ however, process-based training has demon- 
strated comparable effects in older adults and young adults. ${ }^{9}$

Process-based cognitive training targets basic processing capacities, such as speed of processing, working memory, and executive function, that are associated with the efficient operation of higher cognitive function. ${ }^{9}$ This approach aims to exert influence over the mechanisms underlying memory function by training more general cognitive techniques associated with memory processes. Processing capacities are closely related to age-related cognitive decline,${ }^{10}$ and malfunctioning in any of the processing capacities can lead to poor memory performance. According to the literature, process-based interventions were more effective than strategy-based interventions in older adults; in particular, working memory and executive function training was not only effective but also transferable to untrained cognitive domains in this group., ${ }^{9,11,12}$

Delayed free recall, a traditional measure used to assess the effect of cognitive training, is a mixed outcome of the memory processes. According to the Boston Process Approach to neuropsychological assessment, ${ }^{13}$ which emphasizes how an individual performs a task (i.e., process) instead of whether or not he/she succeeds, there can be multiple reasons for poor performance on delayed free recall. These include poor registration due to attention problems, poor consolidation due to hippocampal pathology, and inefficient search for retrieval that reflects frontal lobe dysfunction. ${ }^{14,15}$ Poor delayed recall can also result from interference by irrelevant or similar stimuli if participants demonstrate intrusion errors or reduced recall after disturbing stimuli. Therefore, this complex measure is insufficient for detecting cognitive changes that result from process-based cognitive training aiming to improve mechanisms underlying memory.

In this study, we investigated the effects of process-based cognitive training that targets working memory and cognitive control on memory improvement in healthy elderly adults and patients with MCI. Scores and indexes that reflect underlying memory processes were used to assess which areas were influenced by working memory and cognitive control training. We further sought to investigate whether the effects of process-based training in MCI patients were comparable to those in healthy elderly adults, since there may be different causes of memory dysfunction in healthy older adults and MCI patients.

\section{METHODS}

\section{Participants}

Forty healthy elderly subjects and 40 patients with MCI between 55 and 85 years of age were each randomly assigned to one of two groups: intervention or control. Healthy elderly subjects included individuals who were between 55 and 85 years of age and had not been diagnosed with MCI or any kind of dementia. MCI subjects included individuals who were between 55 and 85 years of age and met the MCI criteria suggested by the International Working Group on MCI. ${ }^{16} \mathrm{We}$ excluded individuals who had any neurological or psychiatric disease, were clinically suspected to have other medical conditions that could affect cognitive function, or had visual or hearing problems that could affect their performance on computerized cognitive training.

\section{Procedure}

The study was approved by the Institutional Review Board of Samsung Medical Center (No. 2015-10-080, No. 2016-01013). All participants provided written informed consent in accordance with the Declaration of Helsinki. Each subject was randomly assigned by computerized randomization (https:// www.randomization.com) to one of two groups: intervention ( $n=40,20$ healthy elderly subjects and $20 \mathrm{MCI}$ patients) or control ( $n=40,20$ healthy elderly subjects and 20 MCI patients). Of the 80 participants, eleven participants left the study before completion: six participants (3 normal elderly subjects and $1 \mathrm{MCI}$ patient) from the control group and five participants (3 normal elderly subjects, 2 MCI patients) from the intervention group. These individuals either refused to undergo the intervention procedure or were lost to follow-up after the pre-intervention assessment. Data obtained from sixty-nine participants who completed the study were analyzed. The intervention group underwent cognitive training for 30 minutes per day, three times per week, for four consecutive weeks. The control group did not undergo training. To assess the effectiveness of the cognitive training, comprehensive neuropsychological tests, including memory tests, were administered three times: baseline (at the beginning of the study), after the training intervention (i.e., at four weeks), and at a four-week follow-up visit (i.e., eight weeks after the beginning of the study) in both the intervention and control groups. The study timeline is depicted in Figure 1. Table 1 shows demographic variables for each group. There were no differences in the mean age, years of education, or sex ratio between the intervention and control groups.

\section{Interventions}

The subjects assigned to the intervention group visited the hospital three times per week and underwent computerized cognitive training for 30 minutes under the supervision of an occupational therapist for four weeks. All subjects were trained for a total of six hours during the four-week intervention period.

\section{Computerized cognitive training}

'Younger travel ${ }^{\mathbb{R}}$ ' is a computerized cognitive training pro- 


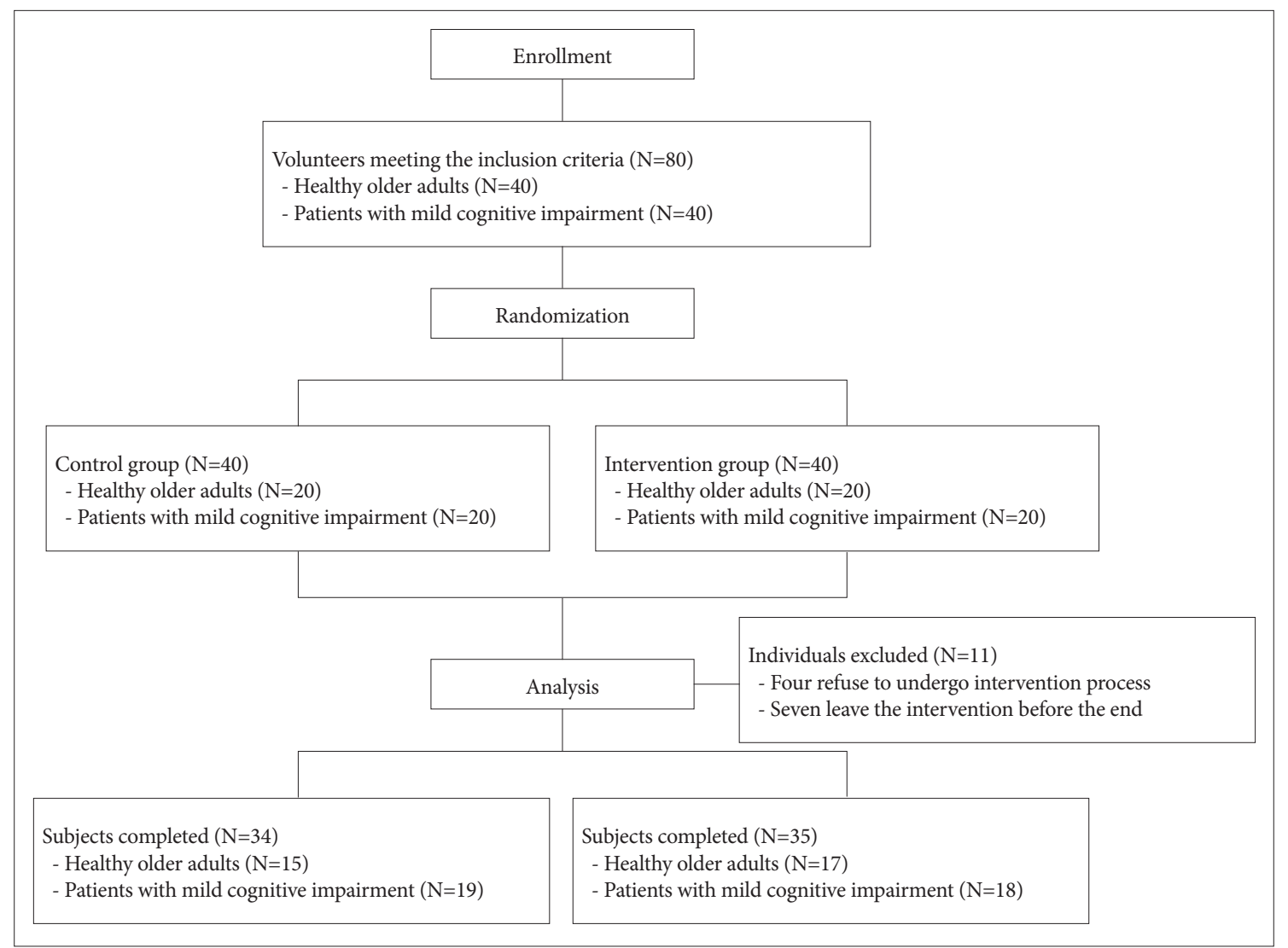

Figure 1. A flow diagram of the study.

Table 1. Demographic variables of participants in control and experimental (training) groups

\begin{tabular}{|c|c|c|c|c|c|c|c|}
\hline & \multicolumn{3}{|c|}{ Control group $(\mathrm{N}=34)$} & \multicolumn{3}{|c|}{ Experimental (training) group $(\mathrm{N}=35)$} & \multirow{2}{*}{$t / \chi^{2}$} \\
\hline & All $(\mathrm{N}=34)$ & Healthy $(\mathrm{N}=15)$ & MCI $(\mathrm{N}=19)$ & All $(\mathrm{N}=35)$ & Healthy $(\mathrm{N}=17)$ & $\mathrm{MCI}(\mathrm{N}=18)$ & \\
\hline Age, M (SD) & $65.44(8.10)$ & $61.33(6.49)$ & $68.68(7.90)$ & $65.31(7.47)$ & $62.00(6.50)$ & $68.44(7.11)$ & 0.068 \\
\hline Education, M (SD) & $11.71(3.72)$ & $12.60(2.53)$ & $11.00(4.38)^{*}$ & $13.26(3.47)$ & $12.71(3.33)$ & $13.78(3.61)^{*}$ & -1.792 \\
\hline Sex, M:F & $8: 26$ & $4: 11$ & $4: 15$ & $9: 26$ & 3:14 & $6: 12$ & 0.044 \\
\hline
\end{tabular}

No significant differences between the control and cognitive training groups were observed. *significant difference $(\mathrm{p}<0.05)$ between the MCI control and MCI training group. MCI: mild cognitive impairment

gram operable on a tablet and developed by C2Monster Co., Ltd. (Seoul, South Korea) that seeks to improve cognitive function in older individuals and patients with MCI. This program consists of four games targeting the major symptoms of cognitive disorders: cognitive control, working memory, memory, and visuospatial perception. Among these four training games, the cognitive control and working memory training games were used as the intervention tasks. Each game consists of multiple difficulty levels, and all participants started from the lowest level. The trainee leveled up if he/she performed with at least $80 \%$ correct responses; otherwise, the trainee repeated the same level until the criteria were met.

\section{Measures}

The Verbal Learning and Memory Test of the Computer- ized Neurocognitive Function Test (CNT; Maxmedica, Seoul, South Korea) was used as the primary outcome. In this test, participants listened to a list of 15 words (list A) five times (trial A1-A5) via the recorded voice of the computer program and recalled out loud as many words as possible after each trial (immediate recall). After the fifth trial (A5), a new interference list (list B) was presented and recalled. After the presentation of list $B$, participants recalled the words from list $A$ again (A6). After a 20-min delay, participants were asked to recall as many original words from list $\mathrm{A}$ as possible (delayed recall), and they were then asked to select words from list $A$ out of 50 words presented on a computer screen containing 15 list A words (recognition). Seven scores were obtained as outcome measures in this study: 1 ) the total number of words recalled immediately after trials $\mathrm{A} 1$ to $\mathrm{A} 5(\mathrm{~A} 1+\mathrm{A} 2+\mathrm{A} 3+\mathrm{A} 4+$ 
A5; total learning), 2) the difference between A5 and A1 (A5$\mathrm{A} 1$; learning index), 3) the total number of words recalled after a 20-min delay (delayed recall), 4) the total number of words correctly selected from the 50-word list (recognition), 5) the difference between A5 and delayed recall divided by A5 [(A5delayed recall)/A5; percentage of forgetting], 6) the difference between A5 and B divided by A5 [(A5-B)/A5; prospective interference], 7) the difference between $A 5$ and A6 divided by A5 [(A5-A6)/A5; retrospective interference].

Other neurocognitive tests selected from the CNT in addition to memory were also included in this study. The digit/ spatial span forward and backward tests were used to measure auditory or visual attentional capacity and working memory. The auditory/visual continuous performance test was used to measure auditory and visual sustained attention. The visual learning test was used to measure visual recognition. The trail making test and the Stroop color and word test were used to measure the executive aspect of cognitive function, including set shifting, working memory, cognitive control, and inhibition.

The Korean Montreal Cognitive Assessment (K-MoCA) ${ }^{17}$ is the Korean version of the Montreal Cognitive Assessment, ${ }^{18}$ which measures global cognitive function with a total score from five cognitive domains: attention and concentration, executive function, memory, language, and visuo-constructional skills. The total possible score is 30 points.

Finally, the Korean version of the Geriatric Depression Scale Short Form (K-GDS-SF) ${ }^{19,20}$ was used to assess the presence of depressive symptoms in the participants. It consists of 15 questions about depressive symptoms related to negative thoughts about the self and environment, negative feelings, and cognitive dysfunction.

\section{Statistical analysis}

SPSS ver. 19.0 (IBM, Corp., Armonk, NY, USA) ${ }^{21}$ was used for statistical analyses of these data. Descriptive information for the groups was compared using t-tests or chi-squared tests. Repeated-measures analysis of variance (RMANOVA) was used to compare changes in test scores during the entire study period between groups. Analysis of covariance (ANCOVA) was used to compare post-intervention or follow-up test scores between groups while controlling for pre-intervention test scores. To determine whether treatment effects differed between normal elderly subjects and MCI patients, MCI status was added to the analysis and the interaction effect of training with MCI status was tested. The level for significance was set at $\mathrm{p}<0.05$. Further, all statistical analyses were adjusted for the following background variables: age, sex, education.

\section{RESULTS}

\section{Verbal learning and memory test of the CNT}

Seven scores or indexes from the verbal learning and memory tests at three time points are presented in Table 2. The performances of both the intervention and control groups showed trends for improvement, but a significant beneficial effect of training was observed in delayed recognition ( $\mathrm{p}=0.023$, partial $\left.\eta^{2}=0.12\right)$ and retrospective interference indexes $(\mathrm{p}=$ 0.002 , partial $\eta^{2}=0.20$ ) by RMANOVA (Table 3 ). Delayed recognition scores were increased post-intervention (T1) and at follow-up (T2) in the training group, which was not observed in the control group (Figure 2A). The retrospective interference index, for which a lower score reflects better performance, was reduced initially post-intervention (T1) and slightly increased at follow-up (T2) in the training group, while this index was just slightly reduced at $\mathrm{T} 1$ and $\mathrm{T} 2$ in the control group (Figure 2B). ANCOVAs were used to compare post-intervention and follow-up test scores for both the training and control groups while controlling for pre-intervention test scores as a covariate. The training group showed significant improvement in delayed recognition at follow-up $(p=0.013$, partial $\left.\eta^{2}=0.10\right)$ and in retrospective interference index at post-intervention ( $p=0.002$, partial $\left.\eta^{2}=0.20\right)$ compared to the control group. An interaction effect between training and MCI status was observed only in the index of percentage of forgetting at post-intervention ( $\mathrm{p}=0.028$, partial $\eta^{2}=0.08$ ), with healthy older adults increasing more than MCI patients. There was no other significant interaction effect between training and MCI status by RMANOVA or ANCOVA.

\section{Other neurocognitive tests of the CNT}

The digit span forward $\left(\mathrm{p}=0.015\right.$, partial $\left.\eta^{2}=0.13\right)$ and backward $\left(p=0.014\right.$, partial $\left.\eta^{2}=0.13\right)$ tests, which measure attention and working memory, increased after the intervention in the training group and these increases were significantly different from alterations in the control group by RMANOVA (Tables 2 and 3). The training group demonstrated significant improvement in the digit span forward test at follow-up ( $\mathrm{p}=0.001$, partial $\eta^{2}=0.16$ ) and digit span backward test post-intervention ( $\mathrm{p}=0.007$, partial $\eta^{2}=0.12$ ) by ANCOVA controlling for pre-intervention test scores (Tables 2 and 3). No significant interaction effect of training with MCI status was observed. No other measures demonstrated significant effects of training, MCI status, or interaction between training and MCI status in the statistical analyses.

\section{K-MoCA}

The total K-MoCA score, a measure of global cognitive function, was increased post-intervention and remained elevated 


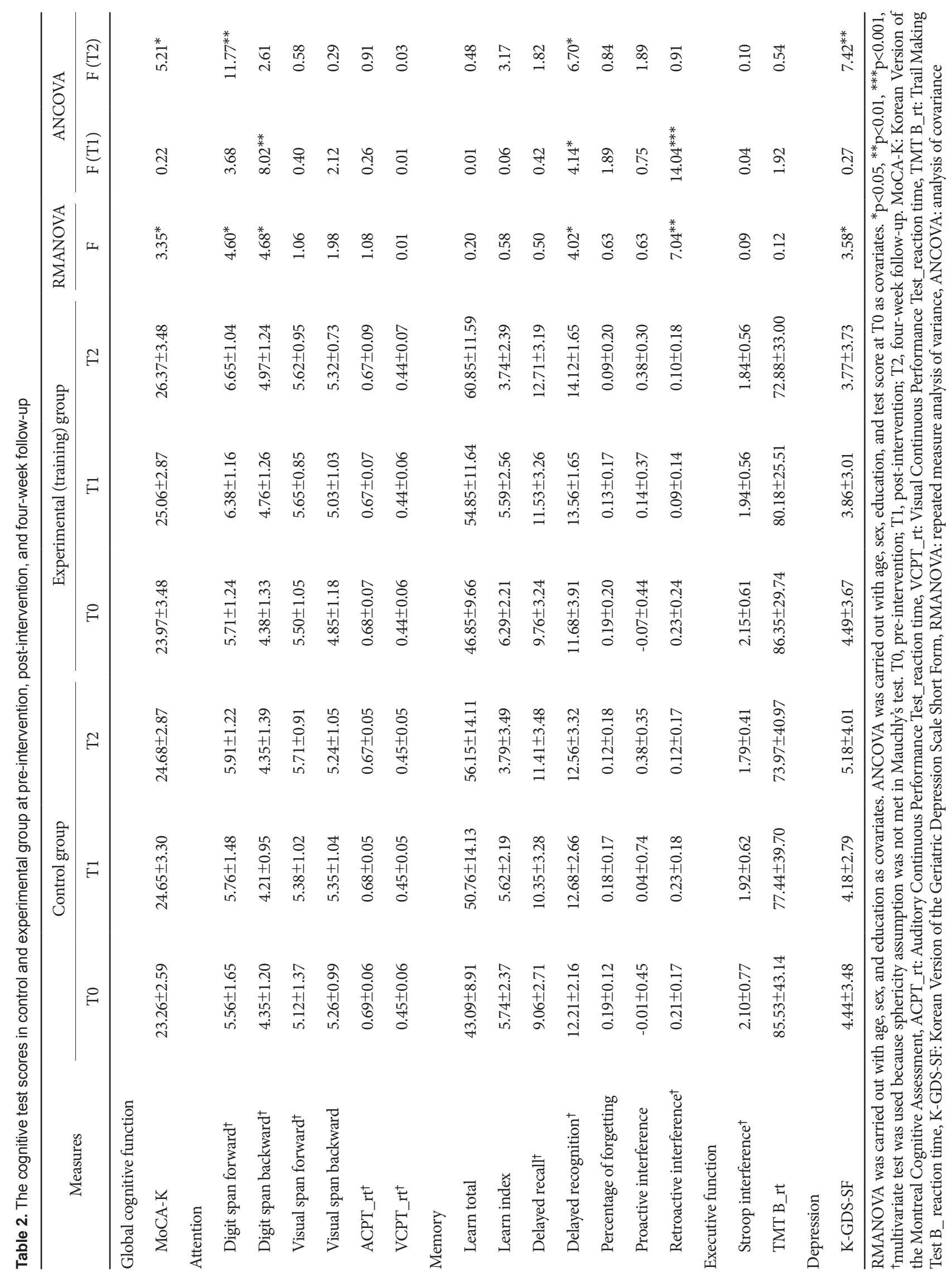




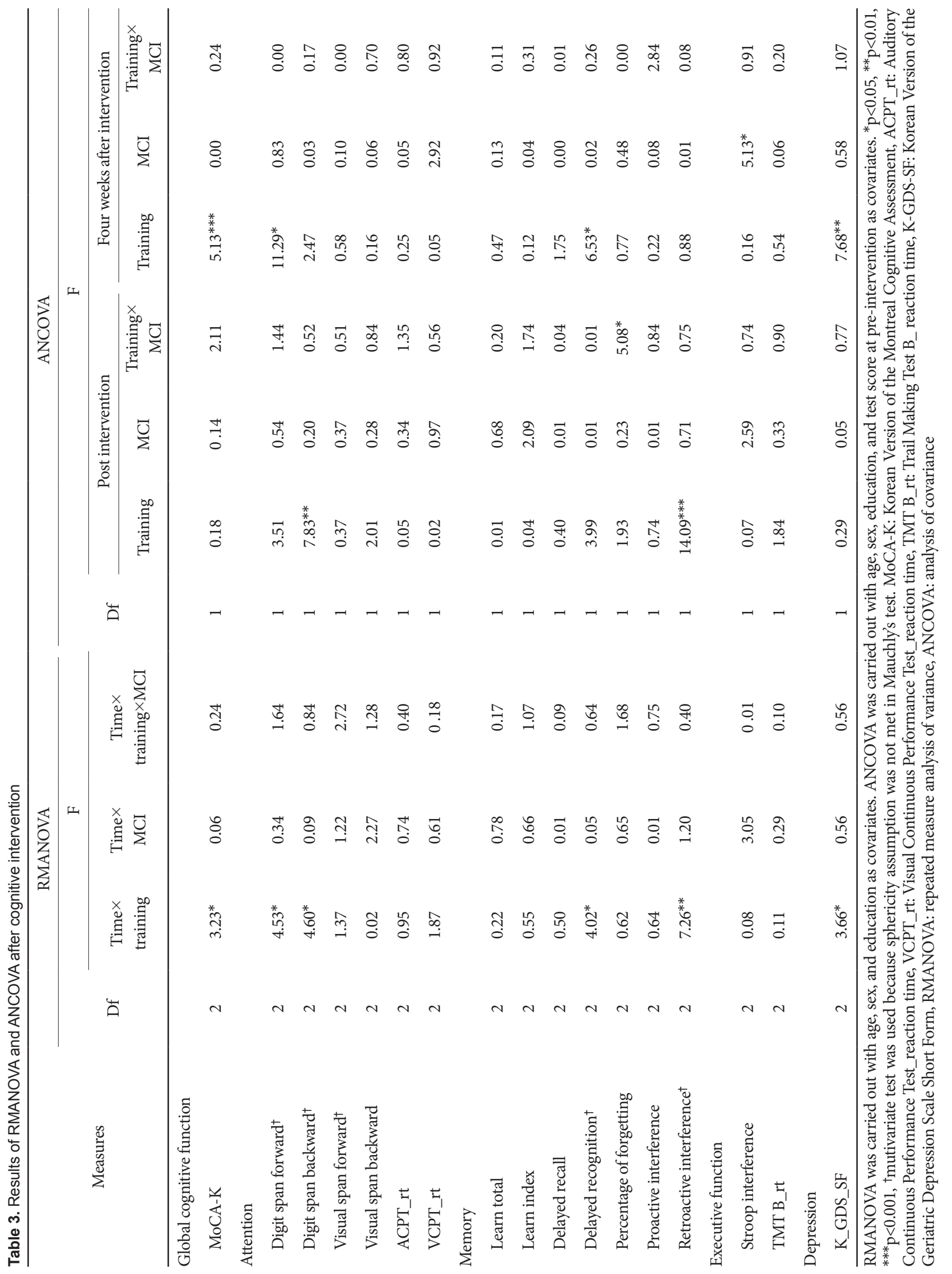


at follow-up in the training group, and these increases were significantly different from the control group by RMANOVA $\left(p=0.043\right.$, partial $\left.\eta^{2}=0.05\right)$ (Tables 2 and 3 ). The results of the ANCOVA showed that the score at follow-up was significantly higher in the training group compared to the control group after controlling for the pre-intervention score ( $\mathrm{p}=0.027$, partial $\eta^{2}=0.08$ ) (Figure $3 \mathrm{~A}$ ). There was no significant interaction effect between training and MCI status by RMANOVA or
ANCOVA analyses.

\section{K-GDS-SF}

Depression was also alleviated post-intervention through follow-up in the training group, and the alteration in scores was significantly different from the control group, where the K-GDS-SF score increased from post-intervention to followup ( $\mathrm{p}=0.028$, partial $\eta^{2}=0.11$ ) (Tables 2 and 3). Compared to the
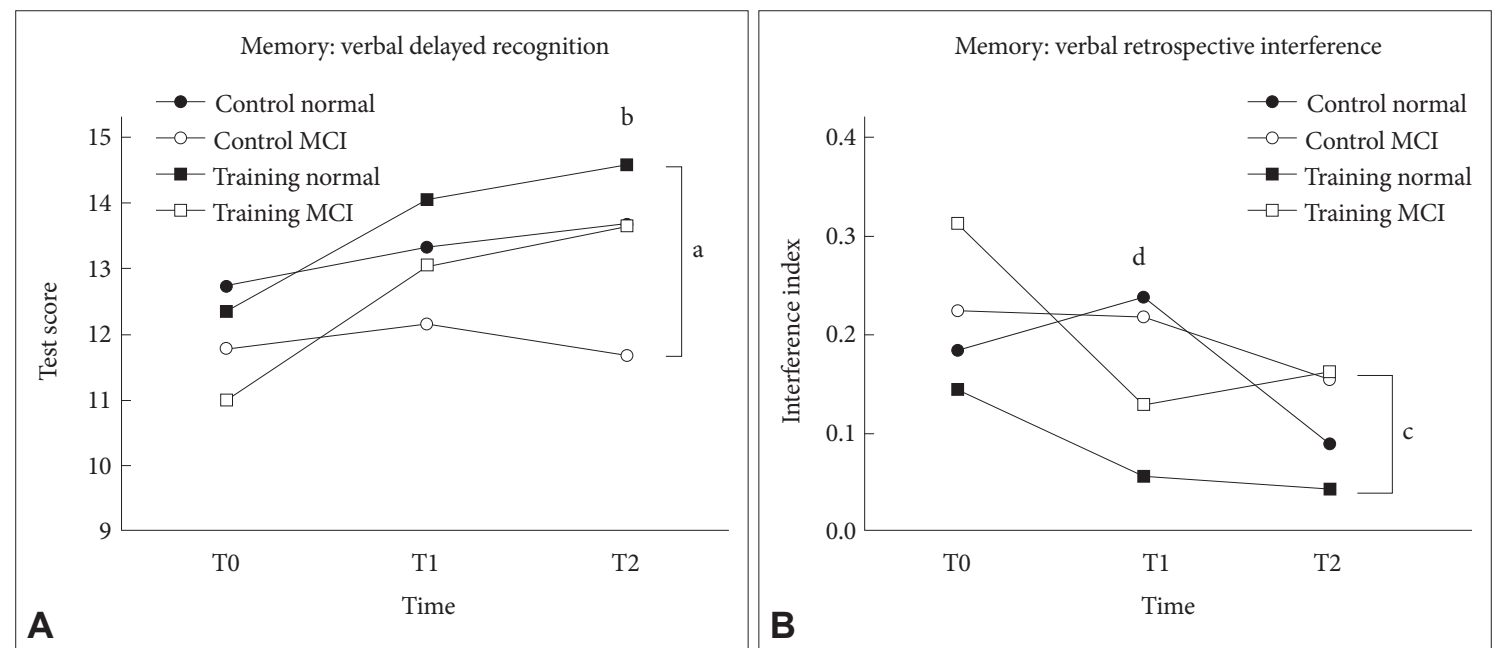

Figure 2. Changes in memory scores after intervention and during follow-up. A: The verbal delayed recognition scores demonstrate the training group showed significant improvement after intervention by RMANOVA $(a, p<0.05)$. An ANCOVA showed that the score at T2 was significantly higher $(b, p<0.05)$ in the training group compared with the control group when T0 was included in the analysis as a covariate. B: The verbal retrospective interference index scores demonstrate the training group showed significant improvement after intervention by RMANOVA $(c, p<0.001)$. An ANCOVA showed that the interference index at $T 1$ was significantly lower $(d, p<0.01)$ in the training group compared with the control group when TO was included in the analysis as a covariate. MCl: mild cognitive impairment, TO: pre-intervention, T1: post-intervention, T2:follow-up, ANCOVA: analysis of covariance, RMANOVA: repeated measure analysis of variance.
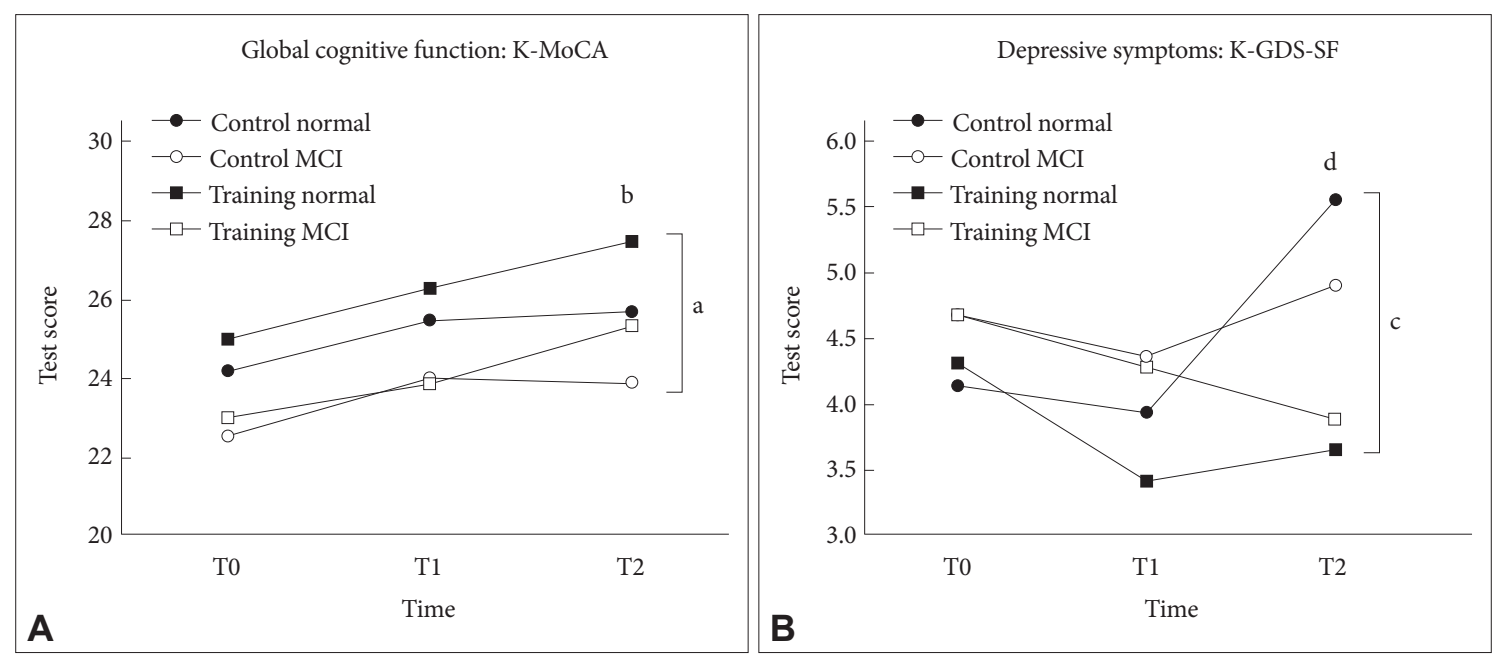

Figure 3. Changes in general cognitive function and depressive symptoms after intervention and during follow-up. A: The general cognitive function scores demonstrate the training group showed significant improvement in Korean Version of the Montreal Cognitive Assessment (KMoCA) scores after intervention by RMANOVA $(a, p<0.05)$. An ANCOVA showed that the K-MoCA score at T2 was significantly higher $(b, p<$ 0.001 ) in the training group compared with the control group when T0 was included in the analysis as a covariate. B: The depressive symptoms scores demonstrate the training group showed significant improvement in Korean Version of the Geriatric Depression Scale Short Form (K-GDS-SF) scores after intervention by RMANOVA (c, p<0.05). An ANCOVA showed that the K-GDS-SF score at T2 was significantly lower $(d, p<0.01)$ in the training group compared with the control group when T0 was included in the analysis as a covariate. MCl: mild cognitive impairment, K-MoCA; Korean Montreal Cognitive Assessment, K-GDS-SF: Korean version of the Geriatric Depression Scale Short Form, TO: preintervention, T1: post-intervention, T2: follow-up, ANCOVA: analysis of covariance, RMANOVA: repeated measure analysis of variance. 
control group, follow-up K-GDS-SF scores were significantly lower in the training group by ANCOVA controlling for preintervention K-GDS-SF score ( $\mathrm{p}=0.007$, partial $\eta^{2}=0.11$ ) (Figure $3 \mathrm{~B}$ ). There was no significant interaction effect between training and MCI status by RMANOVA or ANCOVA.

\section{DISCUSSION}

This study was conducted to investigate the effect of a process-based cognitive intervention that targeted working memory and cognitive control on the improvement of memory in healthy elderly subjects and MCI patients. Cognitive training affected cognitive processes underlying memory function, as evidenced by a reduction in interference by irrelevant stimuli and an increase in recognition of the stored stimuli. Furthermore, it ameliorated depressive symptoms and improved global cognitive function. Finally, these training effects were not moderated by MCI status. Taken together, our results support previous studies demonstrating the effect of cognitive improvement after cognitive training. ${ }^{7,22,23}$ More importantly, they also provide insight into the mechanisms influenced by process-based cognitive training that targets working memory and cognitive control.

In memory tests, the training group showed improvement in their retrograde interference index after cognitive training. A lower retrograde interference index means that recall of learned information (List A) is less disturbed by other similar information (List B) presented thereafter. This process requires the 'central executive' of the Baddeley's working memory system $^{24}$ to allocate attentional resources and regulate cognitive processes to make sure short-term store is actively working for old information (List A) while the 'phonological loop' is rehearsing new information (List B). Cognitive control is another process required for successful memory retrieval from short-term storage, where old information competes with new and irrelevant information. Therefore, in the training group, retrograde interference seemed to be improved through the enhancement of working memory and/or cognitive control after training. In daily life, interference is thought to be one of the reasons for memory loss, which is regarded as an early sign of cognitive aging and dementia. ${ }^{25-27}$ Thus, this result suggests that cognitive training that targets working memory and cognitive control improves memory function by reducing interference not only in normal aging but also in pathological aging.

Participants in the training group showed improvement in the delayed recognition test compared to the control group. This result is consistent with previous reviews reporting that cognitive training improved recognition memory. ${ }^{28}$ Also, there is literature that suggests cognitive control is closely related to the process underlying recognition..$^{29,30}$ One of the numerous factors that can affect memory retrieval is the quality of original memory encoding. ${ }^{31}$ Reduced retrograde interference could contribute to strong and clear encoding of the stimuli, making it less likely for retrieval to be interrupted by similar stimuli presented later. However, although scores increased following intervention in this study, improvement in the delayed free recall score was not statistically significant: this result is not consistent with previous studies. ${ }^{28}$ These two types of retrieval modes, recall and recognition, overlap in part but are associated with different cognitive processes and neural systems. Recognition is associated with the function of the frontal lobe, while recall is more associated with the medial temporal lobe, particularly the hippocampus. ${ }^{14,15}$ Therefore, recognition could benefit more from training that targets cognitive processes associated with the frontal lobe compared to recall.

Other cognitive test scores, such as those from the digit span forward and backward tests, were also improved after training. This is not surprising given that these tests are directly related to the training domain. Remarkably, improvement was observed in general cognitive function after training, as measured with the K-MoCA scale, which suggests a generalized effect of process-based cognitive training. However, it is also possible that the overall score increased because the K-MoCA scale includes tests that assess attention, working memory, and executive function.

Depression was also ameliorated after training, and this effect persisted at follow-up four weeks after intervention. These results differ from those of a previous study ${ }^{23}$ that used non process-based cognitive training, in which cognitive training did not have any effect on the participants' mood. Investigators generally regard depression as a risk factor for cognitive impairment, ${ }^{32,33}$ but on the contrary the results of this study demonstrate that depression could be alleviated by improving cognitive processes such as working memory and cognitive control. Negative thought content $\mathrm{s}^{34,35}$ and negative thought processes $^{36,37}$ are the two factors that contribute to the development of depression. Rumination is a negative thought process that is defined as passive and repetitive thinking of the causes, consequences, and symptoms of the current negative affect, ${ }^{38}$ and it is associated with cognitive control and flexibility. ${ }^{39,40}$ The cognitive training that targeted working memory and cognitive control used in this study might help break the vicious circle of negative thought. This result is consistent with the idea that the dorsolateral prefrontal cortex (DLPFC), which is associated with working memory and cognitive control, provides emotion regulation. ${ }^{41}$

MCI status did not moderate the main effect of cognitive training, indicating that there was no evidence of differential effects of cognitive training between the two populations studied. Poor memory consolidation associated with hippocam- 
pal dysfunction may be one of the major causes of poor memory in MCI patients, considering the similar neurobiological changes seen in amnestic MCI patients and those with Alzheimer's disease. ${ }^{42-44}$ However, the MCI patients also benefited from the cognitive training that targeted working memory and cognitive control, not consolidation. These results suggest that there are various cognitive processes that cognitive training can target to improve memory function, and that even MCI patients can benefit from such cognitive training by focusing training on the preserved functions of the individual patient.

There are several limitations to this study. First, the 'no contact control' used in this study can make the effect of training unclear, because any situation related to cognitive training, including the placebo effect, as well as the training content itself could affect outcome measures. Also, the subjects in the training group could have known that they were being trained due to experiencing repetitive training sessions, though they were not informed that they were in the training group. Therefore, it is not possible to completely exclude the possibility that factors other than the training affected the results as suggested in a previous study. ${ }^{45}$ However, the improvements in memory processes in accordance with the targets of the cognitive training and the observed changes in depressed mood in contrast with the results of a previous study using non processbased cognitive training ${ }^{23}$ support the causal relationship between process-based training and cognitive or emotional improvement. Second, follow-up was performed for only four weeks in this study; we could not assess the long-term maintenance of the training effects. Some outcome measures demonstrated a lagged training effect that showed improvements in follow-up assessment rather than the post-intervention assessment. The lagged effect was reported in a previous study that was conducted for ten years, in which the transfer effect was found in the training group five years later. ${ }^{46,47}$ This result implies that a training effect can be maintained for a considerable period of time. Thus, had the follow-up period been longer, we might have seen longer maintenance or a lagged effect of cognitive training. The potential long-term effects of this process-based cognitive training on cognitive functioning or depression need to be investigated in a future study. Third, the small sample size in our study may have reduced its statistical power and may limit the generalizability of the results, although we tried to meet the minimum requirement for parametric statistics suggested in the literature. ${ }^{48}$ These results should be replicated in further studies with larger samples. Fourth, depression and cognitive function are so closely interrelated that we can not overlook the possibility that depression influenced the training effect. We tried to control the confounding variables, including depression, by randomizing subjects into two groups. The baseline K-GDS-SF score did not differ between the intervention and control groups, thus, it is unlikely that depression affected the baseline cognitive function of the two groups differently. However, we can not exclude the possibility that amelioration of depression due to cognitive training also affected on improvement of cognitive function. This issue should be addressed in further studies. Lastly, the ultimate clinical goal of cognitive training is an improvement in functions of daily living; however, our results have limited generalization to everyday tasks.

This study revealed that process-based cognitive training which targets working memory and cognitive control was effective in improving memory processes including retrograde interference and recognition, and depressive symptoms associated with both normal and pathological aging.

Cognitive training is a safe and alternative option to chemical modulation such as medication for patients with cognitive decline. However, the exact mechanisms by which training works have not been thoroughly elucidated. This study provides insight into these mechanisms by assessing a wide range of scores and indexes that represent memory processes. In addition, this study provided evidence that only six hours of short-term intervention was sufficient to demonstrate a positive effect due to cognitive training. These results may help guide healthcare providers in establishing intervention plans for patients with cognitive impairment.

\section{Acknowledgments}

This work was supported by Institute for Information \& Communications Technology Promotion (IITP) grant funded by the Korea government (MSIT) (Development for Humancare Contents) and Korea Evaluation Institute of Industrial Technology (KEIT) grant funded by the Korean government (MOTIE) (No.10067221).

\section{Conflicts of Interest}

The authors have no potential conflicts of interest to disclose.

\section{Author Contributions}

Conceptualization: Minyoung Shin, Ahee Lee, Yun-Hee Kim. Data curation: Ahee Lee, A Young Cho, Minam Son. Formal analysis: Minyoung Shin. Funding acquisition: Yun-Hee Kim. Investigation: Minyoung Shin, Ahee Lee. Methodology: Minyoung Shin, Ahee Lee, A Young Cho, Minam Son. Project administration: Yun-Hee Kim. Resources: Yun-Hee Kim. Software: Minyoung Shin, Ahee Lee, Yun-Hee Kim. Supervision: Yun-Hee Kim. Validation: Minyoung Shin, Ahee Lee, Yun-Hee Kim. Visualization: Minyoung Shin. Writing_original draft: Minyoung Shin. Writing_review \& editing: Yun-Hee Kim.

\section{ORCID iDs}

$\begin{array}{ll}\text { Minyoung Shin } & \text { https://orcid.org/0000-0002-9742-9510 } \\ \text { Ahee Lee } & \text { https://orcid.org/0000-0002-8150-2170 } \\ \text { A Young Cho } & \text { https://orcid.org/0000-0003-3350-6158 } \\ \text { Minam Son } & \text { https://orcid.org/0000-0002-4442-5989 } \\ \text { Yun-Hee Kim } & \text { https://orcid.org/0000-0001-6101-8851 }\end{array}$




\section{REFERENCES}

1. Jessen F, Wolfsgruber S, Wiese B, Bickel H, Mösch E, Kaduszkiewicz H, et al. $\mathrm{AD}$ dementia risk in late $\mathrm{MCI}$, in early $\mathrm{MCI}$, and in subjective memory impairment. Alzheimers Dement 2014;10:76-83.

2. Petersen RC. Mild cognitive impairment as a diagnostic entity. J Intern Med 2004;256:183-194.

3. Salthouse TA. What and when of cognitive aging. Curr Direct Psychol Sci 2004;13:140-144.

4. Verhaeghen P, Marcoen A, Goossens L. Improving memory performance in the aged through mnemonic training: a meta-analytic study. Psychol Aging 1992;7:242-251.

5. Cavallini E, Pagnin A, Vecchi T. Aging and everyday memory: the beneficial effect of memory training. Arch Gerontol Geriatr 2003;37:241-257.

6. Gross AL, Parisi JM, Spira AP, Kueider AM, Ko JY, Saczynski JS, et al. Memory training interventions for older adults: a meta-analysis. Aging Ment Health 2012;16:722-734.

7. Hertzog C, Kramer AF, Wilson RS, Lindenberger U. Enrichment effects on adult cognitive development: can the functional capacity of older adults be preserved and enhanced? Psychol Sci Public Interest 2008;9: $1-65$.

8. Verhaeghen P, Marcoen A. On the mechanisms of plasticity in young and older adults after instruction in the method of loci: evidence for an amplification model. Psychol Aging 1996;11:164-178.

9. Karbach J, Verhaeghen P. Making working memory work: a meta-analysis of executive-control and working memory training in older adults. Psychol Sci 2014;25:2027-2037.

10. Li SC, Lindenberger U, Hommel B, Aschersleben G, Prinz W, Baltes PB. Transformations in the couplings among intellectual abilities and constituent cognitive processes across the life span. Psychol Sci 2004;15: 155-163.

11. Buschkuehl M, Jaeggi SM, Hutchison S, Perrig-Chiello P, Däpp C, Müller $\mathrm{M}$, et al. Impact of working memory training on memory performance in old-old adults. Psychol Aging 2008;23:743-753.

12. Brehmer $Y$, Westerberg H, Bäckman L. Working-memory training in younger and older adults: training gains, transfer, and maintenance. Front Hum Neurosci 2012;6:63.

13. Milberg WP, Hebben N, Kaplan E, Grant I Adams K. The Boston Process Approach to Neuropsychological Assessment. In: Grant I, Adams K, Editors. Neuropsychological Assessment of Neuropsychiatric and Neuromedical Disorders. New York: Oxford University Press, 2009, p. 42-65.

14. Squire LR. Memory and the hippocampus: a synthesis from findings with rats, monkeys, and humans. Psychol Rev 1992;99:195-231.

15. Zola-Morgan SM, Squire LR. The primate hippocampal formation: evidence for a time-limited role in memory storage. Science 1990;250:288290.

16. Winblad B, Palmer K, Kivipelto M, Jelic V, Fratiglioni L, Wahlund LO, et al. Mild cognitive impairment-beyond controversies, towards a consensus: report of the International Working Group on Mild Cognitive Impairment. J Intern Med 2004;256:240-246.

17. Lee JY, Lee DW, Cho SJ, Na DL, Jeon HJ, Kim SK, et al. Brief screening for mild cognitive impairment in elderly outpatient clinic: validation of the Korean version of the Montreal Cognitive Assessment. J Geriatr Psychiatry Neurol 2008;21:104-110.

18. Nasreddine ZS, Phillips NA, Bédirian V, Charbonneau S, Whitehead V, Collin I, et al. The Montreal Cognitive Assessment, MoCA: a brief screening tool for mild cognitive impairment. J Am Geriatr Soc 2005; 53:695-699.

19. Sheikh JI, Yesavage JA. Geriatric Depression Scale (GDS): recent evidence and development of a shorter version. Clin Gerontol 1986;5:165173

20. Bae JN, Cho MJ. Development of the Korean version of the Geriatric Depression Scale and its short form among elderly psychiatric patients. J Psychosom Res 2004;57:297-305.
21. IBM. IBM SPSS Statistics for Windows Version 19.0. New York: IBM Corp; 2010.

22. Rebok GW, Ball K, Guey LT, Jones RN, Kim HY, King JW, et al. Ten-year effects of the advanced cognitive training for independent and vital elderly cognitive training trial on cognition and everyday functioning in older adults. J Am Geriatr Soc 2014;62:16-24.

23. Günther VK, Schäfer P, Holzner B, Kemmler G. Long-term improvements in cognitive performance through computer-assisted cognitive training: a pilot study in a residential home for older people. Aging Ment Health 2003;7:200-206.

24. Baddeley AD, Hitch G. Working Memory. In: Bower GH, Editor. Psychology of Learning and Motivation. New York: Elsevier, 1974, p.47-89.

25. Davis KL, Price CC, Kaplan E, Libon DJ. Error analysis of the nine-word California Verbal Learning Test (CVLT-9) among older adults with and without dementia. Clin Neuropsychol 2002;16:81-89.

26. Multhaup KS, Balota DA, Faust ME. Exploring semantic memory by investigating buildup and release of proactive interference in healthy older adults and individuals with dementia of the Alzheimer type. J Int Neuropsychol Soc 2003;9:830-838.

27. Ebert PL, Anderson ND. Proactive and retroactive interference in young adults, healthy older adults, and older adults with amnestic mild cognitive impairment. J Int Neuropsychol Soc 2009;15:83-93.

28. Kelly ME, Loughrey D, Lawlor BA, Robertson IH, Walsh C, Brennan S. The impact of cognitive training and mental stimulation on cognitive and everyday functioning of healthy older adults: a systematic review and meta-analysis. Ageing Res Rev 2014;15:28-43.

29. Hussey EK, Harbison J, Teubner-Rhodes SE, Mishler A, Velnoskey K, Novick JM. Memory and language improvements following cognitive control training. J Exp Psychol Learn 2017;43:23-58.

30. Jacoby LL, Shimizu Y, Daniels KA, Rhodes MG. Modes of cognitive control in recognition and source memory: depth of retrieval. Psychon Bull Rev 2005;12:852-857.

31. Tulving E, Thomson DM. Encoding specificity and retrieval processes in episodic memory. Psychol Rev 1973;80:352-373.

32. Modrego PJ, Ferrández J. Depression in patients with mild cognitive impairment increases the risk of developing dementia of Alzheimer type: a prospective cohort study. Arch Neurol 2004;61:1290-1293.

33. Geerlings MI, Bouter L, Schoevers R, Beekman A, Jonker C, Deeg D, et al. Depression and risk of cognitive decline and Alzheimer's disease: results of two prospective community-based studies in The Netherlands. Br J Psychiatry 2000;176:568-575.

34. Hertel PT, Gerstle M. Depressive deficits in forgetting. Psychol Sci 2003; 14:573-578.

35. Gotlib IH, Krasnoperova E, Yue DN, Joormann J. Attentional biases for negative interpersonal stimuli in clinical depression. J Abnorm Psychol 2004;113:127-135.

36. Nolen-Hoeksema S, Wisco BE, Lyubomirsky S. Rethinking rumination. Perspect Psychol Sci 2008;3:400-424.

37. Wisco BE, Gilbert KE, Marroquín B. Maladaptive processing of maladaptive content: rumination as a mechanism linking cognitive biases to depressive symptoms. J Exp Psychopathol 2014;5:329-350.

38. Nolen-Hoeksema S. Responses to depression and their effects on the duration of depressive episodes. J Abnorm Psychol 1991;100:569-582.

39. De Lissnyder E, Koster EH, Goubert L, Onraedt T, Vanderhasselt MA, De Raedt R. Cognitive control moderates the association between stress and rumination. J Behav Ther Exp Psychiatry 2012;43:519-525.

40. Davis RN, Nolen-Hoeksema S. Cognitive inflexibility among ruminators and nonruminators. Cogn Ther Res 2000;24:699-711.

41. Davidson RJ. Affective style, psychopathology, and resilience: brain mechanisms and plasticity. Am Psychol 2000;55:1196-1214.

42. De Santi S, de Leon MJ, Rusinek H, Convit A, Tarshish CY, Roche A, et al. Hippocampal formation glucose metabolism and volume losses in MCI and AD. Neurobiol Aging 2001;22:529-539.

43. Fan Y, Batmanghelich N, Clark CM, Davatzikos C, Alzheimer's Disease Neuroimaging Initiative. Spatial patterns of brain atrophy in MCI pa- 
tients, identified via high-dimensional pattern classification, predict subsequent cognitive decline. Neuroimage 2008;39:1731-1743.

44. Caroli A, Testa C, Geroldi C, Nobili F, Guerra UP, Bonetti M, et al. Brain perfusion correlates of medial temporal lobe atrophy and white matter hyperintensities in mild cognitive impairment. J Neurol 2007;254:10001008.

45. Foroughi CK, Monfort SS, Paczynski M, McKnight PE, Greenwood P. Placebo effects in cognitive training. Proc Natl Acad Sci U S A 2016;113: 7470-7474.
46. Ball K, Berch DB, Helmers KF, Jobe JB, Leveck MD, Marsiske M, et al. Effects of cognitive training interventions with older adults. JAMA 2002; 288:2271-2281.

47. Willis SL, Tennstedt SL, Marsiske M, Ball K, Elias J, Koepke KM, et al. Long-term effects of cognitive training on everyday functional outcomes in older adults. JAMA 2006;296:2805-2814.

48. Corder GW, Foreman DI. Nonparametric Statistics for Non-Statisticians. New Jersey: John Wiley \& Sons, Inc.; 2011. 